\title{
Whole Blood Polymerase Chain Reaction in a Neonate with Disseminated Herpes Simplex Virus Infection and Liver Failure
}

\author{
Jennifer A. Scoble, MD ${ }^{1}$ Mark A. Underwood, MD ${ }^{1}$ \\ ${ }^{1}$ Department of Pediatrics, University of California Davis, Sacramento, \\ California \\ Address for correspondence Mark A. Underwood, MD, Ticon 2 Room \\ 237, 2615 Stockton Blvd, Sacramento CA, 95817 \\ Am J Perinatol Rep 2013;3:67-70. \\ (e-mail: mark.underwood@ucdmc.ucdavis.edu).
}

\author{
Abstract \\ Keywords \\ - herpes simplex virus \\ - liver failure \\ - PCR \\ - neonate \\ - disseminated
}

A late preterm neonate born by cesarean section with intact membranes presented at 9 days of life with shock and liver failure. Surface cultures were negative but whole blood polymerase chain reaction was positive for herpes simplex virus type 2 , underscoring the value of this test in early diagnosis of perinatally acquired disseminated herpes simplex virus infection without skin lesions.
A late preterm male neonate was born following a prenatal course that was unremarkable until 10 days prior to delivery when the mother developed a rash on her arm. The following day, she developed a fever to $38.3^{\circ} \mathrm{C}$ and was evaluated with a complete blood count that was normal. The next day, her temperature was $39.4^{\circ} \mathrm{C}$ and she developed headache, neck pain, and myalgias. Her transaminases were elevated and her platelet count was depressed. The mother was admitted to a community hospital and underwent an infectious disease evaluation. She subsequently developed encephalopathy, and an urgent cesarean section was performed at 36 weeks gestation. Rupture of membranes occurred at delivery with clear amniotic fluid. The infant was vigorous with Apgar scores of 8 at 1 minute and 9 at 5 minutes. On postpartum day 2, the mother's herpes simplex virus (HSV)-2 IgM was positive and her HSV-2 IgG was negative. Cervical exam revealed no lesions, but an HSV polymerase chain reaction (PCR) from a cervical swab was positive. A diagnosis of primary HSV viremia in the mother was made. Of note, she was not treated with acyclovir prior to delivery. During admission, a nasal swab was obtained from the infant, and he was sent home on day of life 4 with routine follow-up. The HSV nasal culture was subsequently negative.

The baby remained in good health until 9 days of age when he was sleepier than usual and was having difficulty feeding.
That day, he was brought to his pediatrician's office for a circumcision. He appeared well, so the procedure was performed, and he fed well afterward. That evening he became lethargic then limp, blue, and unresponsive. Rescue breaths were given by the mother and emergency services summoned.

Upon arrival to the community hospital emergency department by ambulance, the infant was limp and jaundiced, with recurrent apnea. Axillary temperature was $31.4^{\circ} \mathrm{C}$. He was intubated and given intravenous saline boluses to total 30 $\mathrm{mL} / \mathrm{kg}$. Laboratory evaluation revealed a white blood cell (WBC) count of $3.5 \times 10^{9} / \mathrm{L}$ with $20 \%$ neutrophils, $3 \%$ bands, and $67 \%$ lymphocytes. C-reactive protein was 1,943 nmol/L. The cerebrospinal fluid (CSF) showed no WBCs and 630 red blood cells per microliter with normal glucose and protein. The lumbar puncture did not yield sufficient CSF for an HSV PCR. Urinalysis was normal. Bacterial cultures of the blood, CSF, and urine and nasal swabs for influenza, respiratory syncytial virus, and pertussis were obtained (all subsequently negative). Ampicillin, gentamicin, and acyclovir were started, and he was transferred to a tertiary facility for further evaluation and treatment.

On arrival to the tertiary hospital, the infant was poorly responsive and hypotonic. His abdomen was soft, with no mass or hepatosplenomegaly. There were no lesions noted on received

October 23, 2012

accepted after revision

February 1, 2013

published online

March 19, 2013
DOI http://dx.doi.org/

10.1055/s-0033-1338167. ISSN 2157-6998.
Copyright $\odot 2013$ by Thieme Medical Publishers, Inc., 333 Seventh Avenue, New York, NY 10001, USA. Tel: +1(212) 584-4662.

\section{License terms}

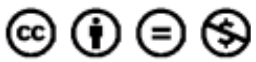




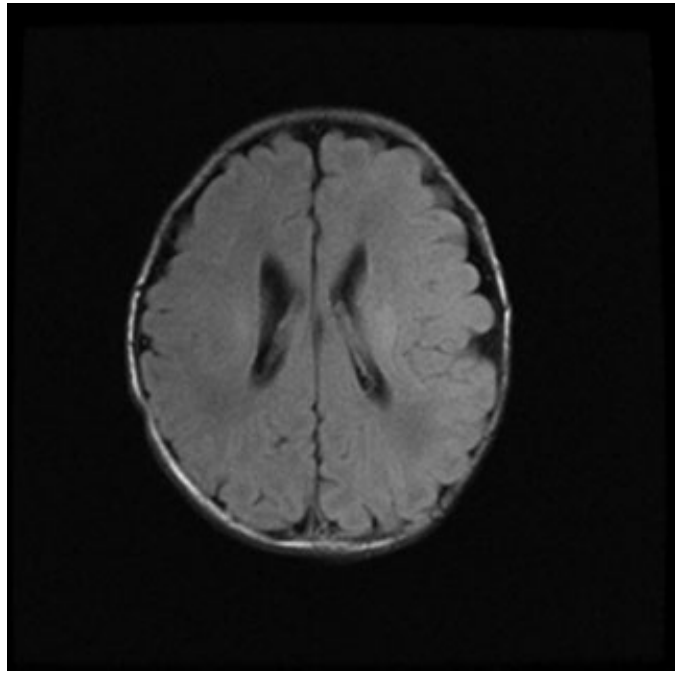

Fig. 1 Magnetic resonance image obtained at day of life 10 demonstrating bilateral areas of periventricular white matter injury.

skin exam. A chest radiograph was unremarkable. Liver enzymes were notable for an aspartate transaminase of $4851 \mathrm{U} / \mathrm{L}$ and alanine transferase (ALT) of $986 \mathrm{U} / \mathrm{L}$. The infant's international normalization ratio was $>11.5$. His platelet count was $14 \times 10^{9} / \mathrm{L}$. Ampicillin and gentamicin were discontinued after 48 hours. Whole blood HSV PCR was positive for HSV-2. Surface cultures of the nasopharynx, anus, and conjunctivae and a urine culture were negative for both HSV1 and HSV-2. Initial serologies for HSV-1 and HSV-2 were negative, but when repeated 6 days later revealed a highly positive IgM to HSV-2. A second lumbar puncture was performed after 17 days of treatment with acyclovir with CSF positive for HSV-2 by PCR. Magnetic resonance imaging of the brain showed periventricular white matter injury (-Fig. 1). An ophthalmologic exam was normal; the baby never developed skin lesions.

The infant's hospital course was significant for liver failure with severe ascites and pleural effusions that ultimately improved over the course of treatment. Laboratory values are summarized in - Table $\mathbf{1}$. His bone marrow recovered as well with normalization of his WBC and platelet counts. He was treated with intravenous acyclovir until his CSF HSV PCR was negative ( 4 weeks) and then was sent home on suppressive oral acyclovir therapy for 6 months with close neurodevelopmental follow-up.

\section{Discussion}

HSV infection in the fetus/neonate is classified as intrauterine infection (either an ascending infection or transplacental infection due to maternal viremia), infection acquired at birth, or infection acquired after birth. Intrauterine infection is much less common and usually presents as a triad of cutaneous, ophthalmologic, and central nervous system (CNS) findings. ${ }^{1}$ HSV infection acquired at birth generally presents between 1 and 2 weeks as disease localized to the skin, eyes, and mouth (40 to $45 \%$ of cases), encephalitis with or without skin disease (30 to $35 \%$ ), or disseminated disease (20 to 25\%). Disseminated disease involves multiple organs, most commonly the liver, lungs, and CNS.

HSV, particularly HSV-1, infection can also be acquired after delivery from direct contact with a mucosal lesion (e.g., contact with lesions on the breast during nursing, being kissed by someone with an oral lesion, or during direct orogenital suctioning as part of ritual circumcision in some orthodox Jewish communities). ${ }^{2}$ The circumcision was not likely related to the infection in this infant given the symptom onset shortly after (or perhaps even prior to) the procedure.

The risk of transmission at delivery is much higher in a woman with a primary HSV infection (25 to $60 \%$ ) than in a woman with a recurrence $(<2 \%)$. This case is instructive in that the mother had an illness consistent with primary HSV viremia/encephalitis but did not have any genital lesions. The mode of acquisition of neonatal HSV was different from the usual intrapartum acquisition as the infant passes through the birth canal. In the latter scenario, delivery by cesarean

Table 1 Laboratory values

\begin{tabular}{|l|l|l|l|l|l|l|l|l|l|}
\hline & DOL 10 & DOL 11 & DOL 12 & DOL 13 & DOL 15 & DOL 16 & DOL 20 & DOL 29 & DOL 36 \\
\hline AST (U/L) & 4951 & 9647 & 4928 & 2427 & 1423 & 344 & 162 & 344 & 133 \\
\hline ALT (U/L) & 986 & 1578 & 973 & 721 & 459 & 359 & 115 & 301 & 113 \\
\hline GGT (U/L) & & & 190 & 171 & 137 & 127 & & 231 & 307 \\
\hline D. bili (mg/dL) & & & 2.4 & 2.8 & 2.9 & 4.1 & 2.2 & 3.4 & 1.5 \\
\hline INR & 11.5 & 2.84 & 2.46 & 1.73 & 2.10 & 1.38 & & 0.93 & \\
\hline BUN (mg/dL) & 16 & 13 & 13 & 8 & 7 & 9 & 13 & 6 & 3 \\
\hline Creatinine (mg/dL) & 0.71 & 0.7 & 0.84 & 0.74 & 0.5 & 0.41 & 0.33 & 0.49 & 0.49 \\
\hline WBC (K/mm $)$ & 2.8 & 4.9 & 10.6 & 19 & 29.2 & 36.8 & 27.8 & 20.2 & 15 \\
\hline HCT (\%) & 44.5 & 43.1 & 38 & 38.2 & 31.6 & 29.8 & 25.3 & 26.2 & 25.3 \\
\hline Platelet (K/mm $\left.{ }^{3}\right)$ & 14 & 38 & 24 & 51 & 21 & 26 & 38 & 262 & \\
\hline
\end{tabular}

Abbreviations: ALT, alanine transferase; AST, aspartate transaminase; BUN, blood urea nitrogen; D. bili, direct bilirubin; DOL, day of life; GGT, gammaglutamyl transpeptidase; HCT, hematocrit; INR, international normalization ratio; WBC, white blood cells. 
section at or shortly after rupture of membranes decreases but does not eliminate risk. The nasal culture obtained after 12 hours in the infant was negative as were the surface cultures obtained at the tertiary hospital. Acyclovir was not started in the first few days of life because the infant looked well, was born by cesarean section, and ultimately had a negative HSV nasal culture. We speculate that whole blood HSV PCR analysis and HSV culture of the conjunctiva, mouth, and anus in addition to the culture of the nasopharynx on the infant at 12 to 24 hours of life may have been helpful in earlier diagnosis. Indeed, given the maternal history of primary multisystem HSV infection and the high risk of transmission to the neonate, one reasonable approach would have been a full diagnostic evaluation as well as starting empiric acyclovir pending laboratory results. This is in marked contrast to an infant born via cesarean section through intact membranes to a mother who has genital herpes without systemic symptoms.

With respect to infants who have been exposed to HSV during the birth process, current guidelines from the American Academy of Pediatrics recommend obtaining swabs of the mouth, nasopharynx, conjunctiva, and anus for HSV culture 12 to 24 hours after delivery for infants born vaginally or by cesarean section to women with active genital HSV lesions (a single swab can be used for all four locations with the anus cultured last). Positive surface cultures obtained after 12 to 24 hours of life suggest active infection, rather than contamination during delivery. ${ }^{3}$ Some experts recommend initiating treatment of the neonate with acyclovir after obtaining the cultures in primary maternal infections but not recurrent infections. If the surface culture is positive, the baby is presumed to be infected; further testing should then include CSF for HSV culture and PCR, whole blood for HSV PCR, and serum ALT. If these further tests are negative, a 10-day course of acyclovir is recommended. ${ }^{3}$

Diagnosis of neonatal HSV is challenging when there are no skin findings. Disseminated or CNS herpes should be considered in all infants presenting in the first 6 weeks of life with severe sepsis without an obvious bacterial cause, unexplained seizures, liver failure, and even fever with irritability plus abnormal CSF. ${ }^{4}$ Testing should include the studies described above (surface cultures, CSF for HSV culture and PCR, whole blood for HSV PCR, and serum ALT). ${ }^{3}$ Empiric acyclovir until HSV infection has been ruled out is reasonable in these situations. ${ }^{5}$ HSV IgM levels in a neonate with birthacquired infection usually do not rise until 2 to 4 weeks after exposure and therefore are not often useful in neonates. ${ }^{3,6}$

Neonatal disseminated HSV infection has high morbidity and mortality even with antiviral treatment. ${ }^{7}$ A recent case series of 11 infants with HSV liver failure reported only two survivors, both of whom were started on acyclovir early. ${ }^{8}$ of note, in this series seven of the infants were diagnosed with infection due to HSV-1 and four infants with HSV-2, highlighting the fact that HSV-1 can cause genital infection in adults and severe disease in the neonate. A second case series of 15 infants with HSV hepatitis suggested the following predictors of more severe disease: positive PCR, lack of skin lesions, and degree of severity of coagulopathy, thrombocytopenia, and cholestasis. ${ }^{9}$ There have been several case reports of successful treatment of fulminant hepatic failure from neonatal HSV with liver transplantation, underscoring the value of early consultation with a liver transplant center. ${ }^{10}$

Poor neurodevelopmental outcomes occur in about $20 \%$ of survivors of disseminated disease and $70 \%$ of those with CNS disease. Neurodevelopmental impairment is unusual in HSV infections presenting with involvement of only the skin, eyes, and mouth. Recurrent skin lesions occur in up to $50 \%$ of survivors of neonatal HSV infection and may be associated with worse developmental outcomes. A recent double-blind placebo-controlled study of 74 infants found that treatment with suppressive acyclovir orally for 6 months after initial 14 to 21 days of parenteral treatment prevented skin recurrences and improved neurodevelopmental outcomes. ${ }^{11}$

The use of quantitative PCR to determine DNA viral load in disseminated herpes has been proposed as a marker of response to therapy in adults. ${ }^{12}$ As in this case, whole blood PCR is a valuable diagnostic tool in neonatal HSV infection; however, data to suggest its value in determining response to therapy in the neonate are lacking. Retrospective diagnosis of neonatal HSV infection has been demonstrated using PCR of blood obtained for routine newborn screening. ${ }^{13}$ The increasing prevalence of HSV-2 infections, the high morbidity and mortality of CNS and disseminated disease, and the challenges of early diagnosis in infants without skin lesions underline the importance of a high index of suspicion in the neonatal period and the need for further research into early detection and improved treatment.

\section{References}

1 Hutto C, Arvin A, Jacobs R, et al. Intrauterine herpes simplex virus infections. J Pediatr 1987;110:97-101

2 Centers for Disease Control and Prevention(CDC). Neonatal herpes simplex virus infection following Jewish ritual circumcisions that included direct orogenital suction-New York City, 2000-2011. MMWR Morb Mortal Wkly Rep 2012;61:405-409

3 Herpes Simplex. In: Pickering L, ed. Red Book: Report of the Committee on Infectious Diseases. 29th ed. Elk Grove Village, IL: American Academy of Pediatrics; 2012

4 Robinson JL, Vaudry WL, Forgie SE, Lee BE. Prevention, recognition and management of neonatal HSV infections. Expert Rev Anti Infect Ther 2012;10:675-685

5 Vanderpluym C, Tawfik G, Hervas-Malo M, Lacaze-Masmonteil T, Kellner J, Robinson JL. Empiric acyclovir for neonatal herpes simplex virus infection. J Matern Fetal Neonatal Med 2012; 25:1278-1282

6 Sullender WM, Miller JL, Yasukawa LL, et al. Humoral and cellmediated immunity in neonates with herpes simplex virus infection. J Infect Dis 1987;155:28-37

7 Kimberlin DW, Lin CY, Jacobs RF, et al; National Institute of Allergy and Infectious Diseases Collaborative Antiviral Study Group. Natural history of neonatal herpes simplex virus infections in the acyclovir era. Pediatrics 2001;108:223-229

8 Verma A, Dhawan A, Zuckerman M, Hadzic N, Baker AJ, MieliVergani G. Neonatal herpes simplex virus infection presenting as acute liver failure: prevalent role of herpes simplex virus type I. J Pediatr Gastroenterol Nutr 2006;42:282-286

9 McGoogan KE, Haafiz AB, González Peralta RP. Herpes simplex virus hepatitis in infants: clinical outcomes and correlates of disease severity. J Pediatr 2011;159:608-611 
70 PCR in Neonatal Disseminated Herpes Scoble, Underwood

10 Egawa H, Inomata Y, Nakayama S, et al. Fulminant hepatic failure secondary to herpes simplex virus infection in a neonate: a case report of successful treatment with liver transplantation and perioperative acyclovir. Liver Transpl Surg 1998;4:513-515

11 Kimberlin DW, Whitley RJ, Wan W, et al; National Institute of Allergy and Infectious Diseases Collaborative Antiviral Study Group. Oral acyclovir suppression and neurodevelopment after neonatal herpes. N Engl J Med 2011;365:1284-1292
12 Beersma MF, Verjans GM, Metselaar HJ, Osterhaus AD, Berrington WR, van Doornum GJ. Quantification of viral DNA and liver enzymes in plasma improves early diagnosis and management of herpes simplex virus hepatitis. J Viral Hepat 2011;18: e160-e166

13 Lewensohn-Fuchs I, Osterwall P, Forsgren M, Malm G. Detection of herpes simplex virus DNA in dried blood spots making a retrospective diagnosis possible. J Clin Virol 2003;26:39-48 